\title{
IDENTIFICAÇÃO DE ÁREAS VULNERÁVEIS AO ALAGAMENTO PLUVIAL NA CIDADE DE NATAL/RN
}

\author{
Matheus Lisboa Nobre da Silva \\ Integrante do Grupo de Pesquisa Mineral do IFRN. \\ E-mail: nobre.mt@gmail.com \\ Mário Tavares de Oliveira Cavalcanti Neto \\ Geólogo, doutorando DINTER Recursos NATURAIS. \\ Professor e coordenador do Grupo de Pesquisa Mineral do IFRN. \\ E-mail:mario@cefetrn.br
}

\section{RESUMO}

Em Natal, capital do estado do Rio Grande do Norte, a impermeabilização do solo e a falta de rede de drenagem em diversos setores da cidade, têm se mostrado como um fator importante para a geração de caos urbano devido aos alagamentos com conseqüências socioeconômicas. Este trabalho se propõe a indicar áreas vulneráveis ao alagamento por águas pluviais, baseados principalmente nas relações topográficas plani-altimétricas e nos fluxos de drenagem urbana. Os resultados foram analisados na perspectiva de proposições que busquem a mitigação deste problema na capital potiguar. Nessa primeira fase foi selecionada uma área no entorno do Campus Central do IFRN. Na continuação, e na dependência de financiamentos, esse estudo será estendido para as demais áreas da cidade.

PALAVRAS-CHAVE: Sensoriamento Remoto, Drenagem, Alagamentos, Meio Ambiente.

\section{IDENTIFICATION OF VULNERABLE AREAS TO PLUVIAL FLOODING IN THE CITY OF NATAL/RN}

In Natal, the state capital of Rio Grande do Norte, the soil sealing and the lack of drainage in several sectors of the city, have been proved as one important factor for the generation of urban caos due to floods with socioeconomic consequences. This paper aims to indicate vulnerable areas to flooding from rainwater based, mainly, on plani-altimetric topographic relations and on urban drainage flows. The results were analysed into the perspective of propositions that seek the decrease of this problem in the city. In this first phase, it was selected an area surrounding the IFRN's Central Campus. In continuation, and depending on financing, this study may be extended to other areas of the city.

KEYWORDS: Remote Sensing, Drainage, Floodings, Environment. 


\section{IDENTIFICAÇÃO DE ÁREAS VULNERÁVEIS AO ALAGAMENTO PLUVIAL NA CIDADE DE NATAL/RN}

\section{INTRODUÇÃO}

Os alagamentos se tornaram um problema bastante recorrente no cotidiano das famílias brasileiras, do interior e das capitais. Essa problemática está diretamente relacionada à intervenção humana na constituição dos espaços urbanos, na redução de áreas verdes, na impermeabilização do solo, no aumento da especulação imobiliária, na contaminação de aquíf́eros, entre outras ações antrópicas decorrentes do uso e ocupação desordenada do solo. Segundo o Manual de Desastres (2003), “os alagamentos são freqüentes nas cidades mal planejadas ou quando crescem explosivamente, dificultando a realização de obras de drenagem e de esgotamento de águas pluviais", sendo, comumente, aliado às enxurradas. Ainda segundo o referido Manual, as principais consequiências dos alagamentos são materiais e humanas.

Estudos relacionados à questão dos alagamentos já foram realizados em algumas cidades do Brasil, a exemplo de João Pessoa (Sobreira et al, 2004) e Rio de Janeiro (Silveira et al, 2008). Ambos os autores tratam da influência do ser humano na intensificação destes eventos nas cidades brasileiras.

A cidade de Natal está situada na meso-região do Litoral Leste Potiguar, onde ocorrem os maiores índices pluviométricos do Estado, conforme atestado pela EMPARN (Empresa de Pesquisa Agropecuária do Rio Grande do Norte) através de monitoramento da pluviosidade dos últimos 10 anos na cidade, obtendo uma média anual de precipitação acima de $1.500 \mathrm{~mm}$. As figuras 1 e 2 tratam, respectivamente, dos volumes pluviométricos mensais e anuais medidos na cidade.

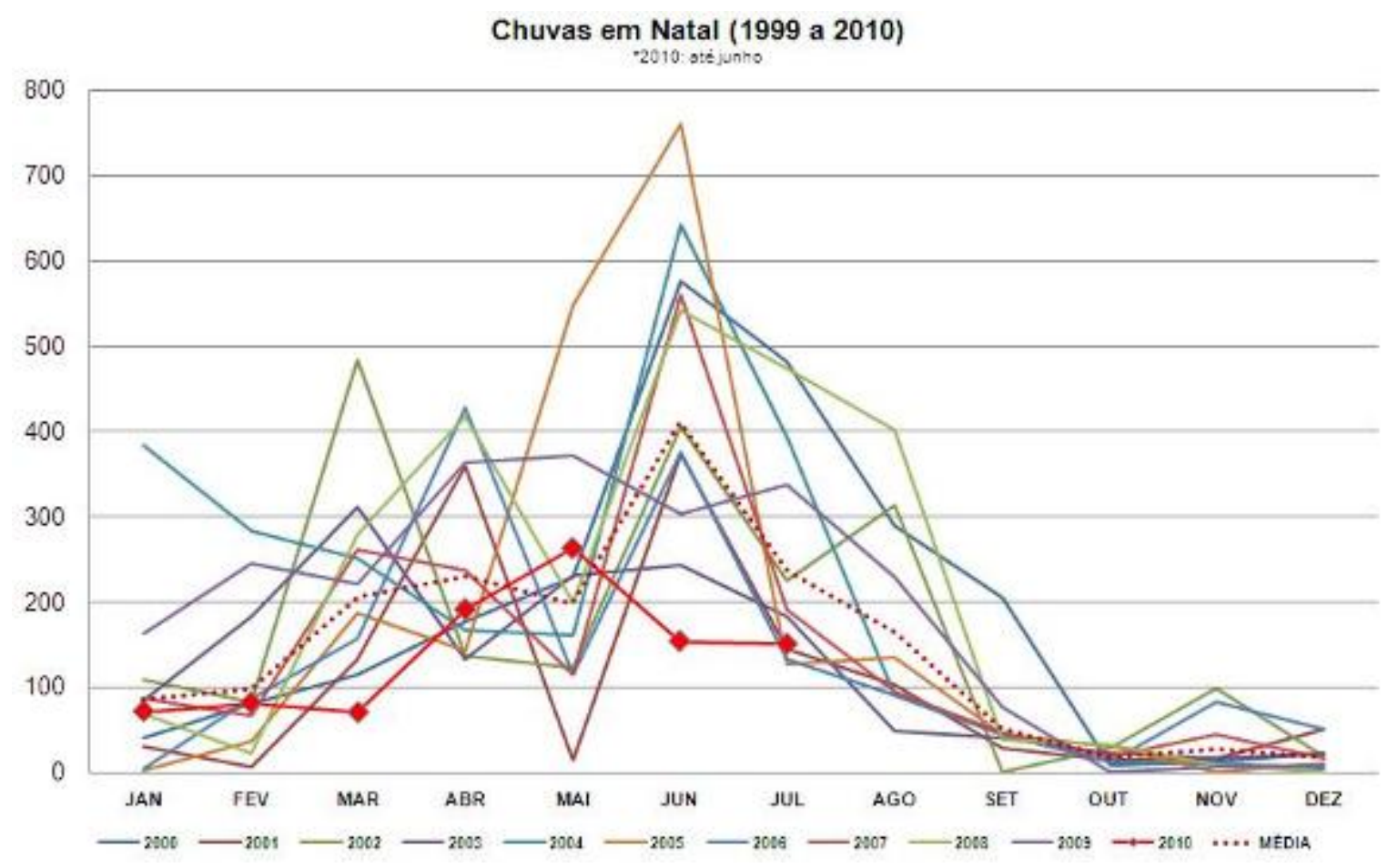

Figura 1 - Volumes pluviométricos mensais em Natal-RN de 1 de jan/1999 a 3 de jul/2010 (compilado da EMPARN, 2010) 


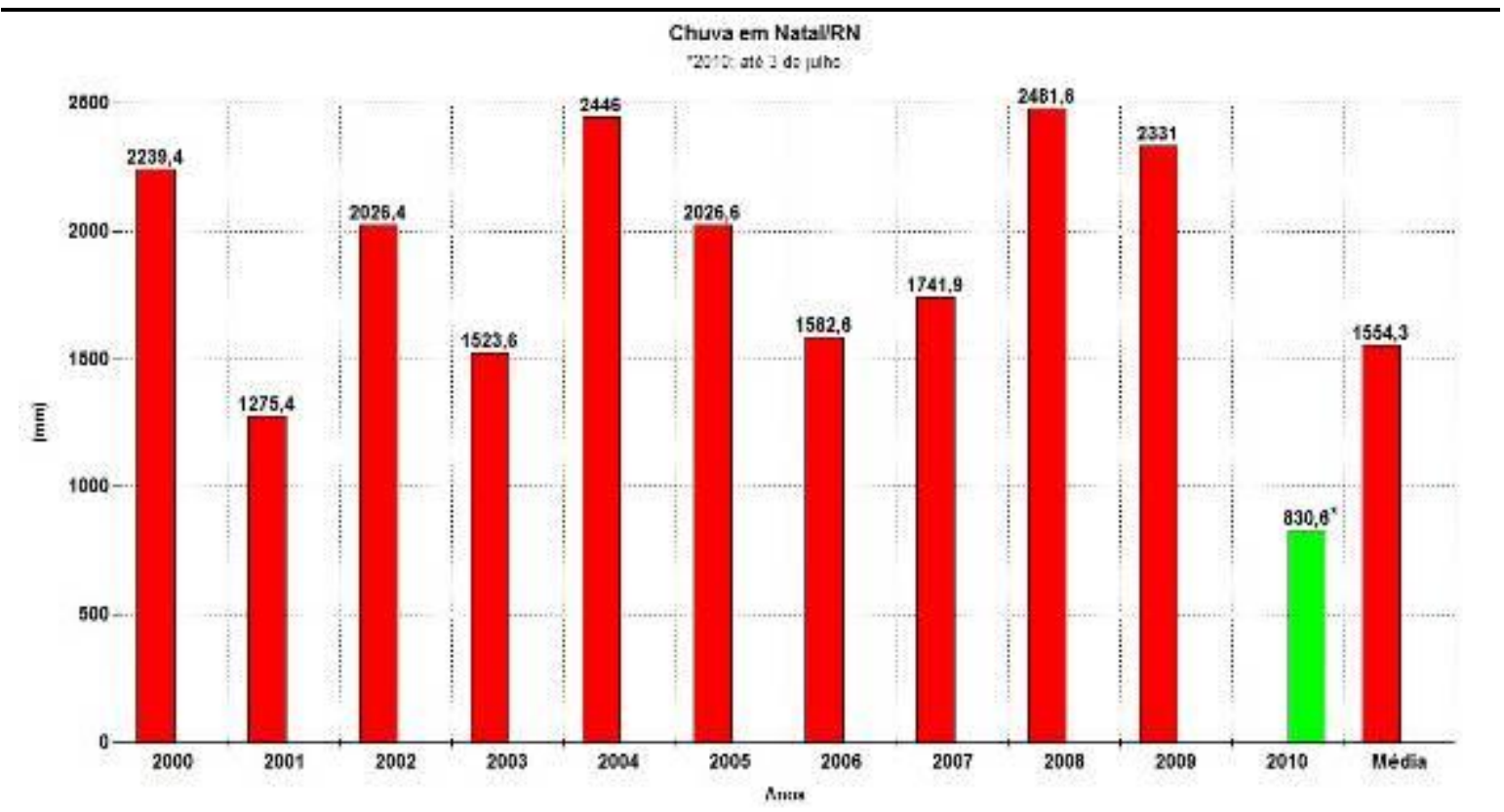

Figura 2 - Volumes pluviométricos anuais em Natal-RN de 1999 a 3 de jul/2010 (compilado da EMPARN, 2010).

Os alagamentos constituem um problema que afeta toda a rede urbana. Mesmo pequenos volumes pluviométricos são capazes de gerar caos nas cidades mal preparadas, exemplo de Natal, em que cenas de caos se propagam pelas vias urbanas em dias chuvosos (vide figura 3). Portanto, na capital potiguar a situação não é diferente das outras cidades do Brasil, podendo ser considerada como alarmante para o cotidiano da população, que muitas vezes é a principal culpada por esse acontecimento. "O desrespeito à legislação, muitas vezes motivado pela especulação imobiliária, o mau uso do solo e o desmatamento têm gerado um quadro de degradação responsável pela potencialização a processos de risco" (Santos, 2007). Este estudo é direcionado na proposição de ações mitigadoras para esse "risco ambiental urbano" (Cortez, 2003).

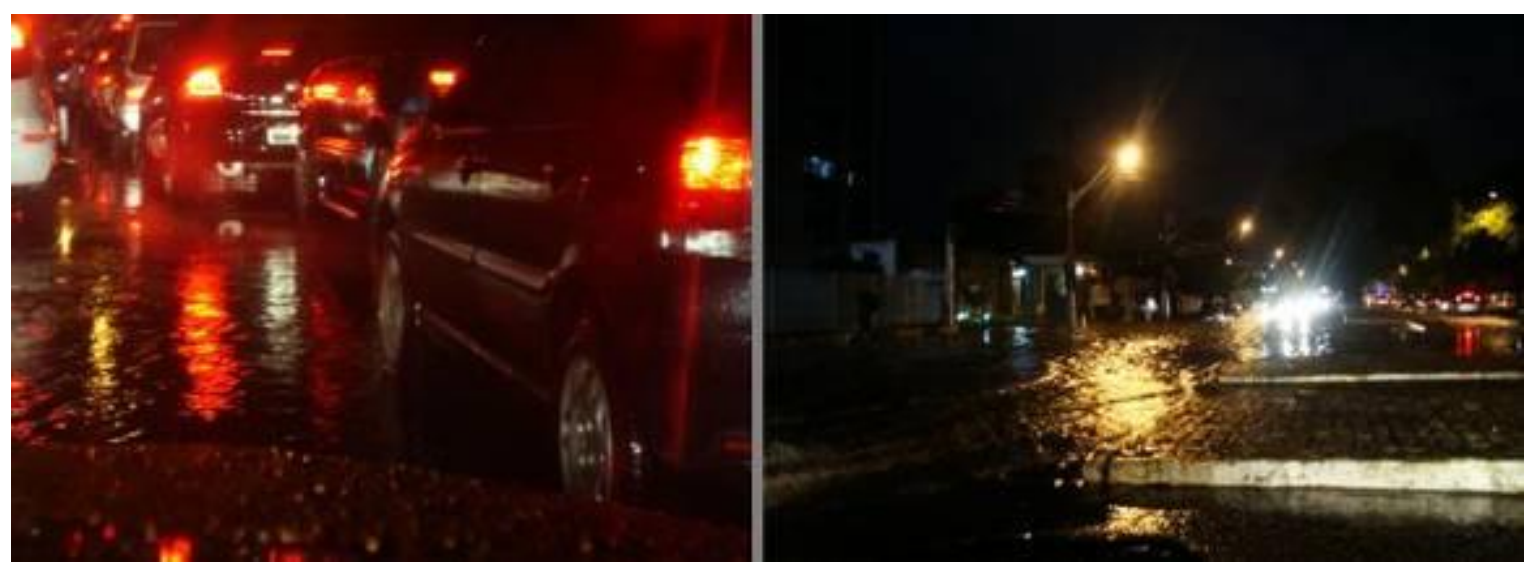

Figura 3 - Cenas de um dia chuvoso na cidade de Natal-RN, 17 de junho de 2010. Volume pluviométrico neste dia, segundo a EMPARN: $12,5 \mathrm{~mm}$ 


\section{RECORTE ESPACIAL DESTE TRABALHO}

A área enfocada neste trabalho, de aproximadamente $3,2 \mathrm{~km}^{2}$, é fragmento de cena englobando o entorno do campus central do IFRN, conforme demonstra a figura 4. Compreende os bairros de Tirol, Lagoa Seca e Lagoa Nova, onde estão sediados importantes setores sócio-econômicos da cidade, com diversos estabelecimentos comerciais, incluindo o principal Shopping Center (Midway Mall), o principal Hospital público (Hospital Walfredo Gurgel) e, na educação, um campus de uma universidade privada, a UNP, e o campus Natal-Central do IFRN como mostrado na figura 5. Nesta área situam-se os bairros mais valorizados da cidade e, provavelmente, o metro quadrado mais caro de Natal.

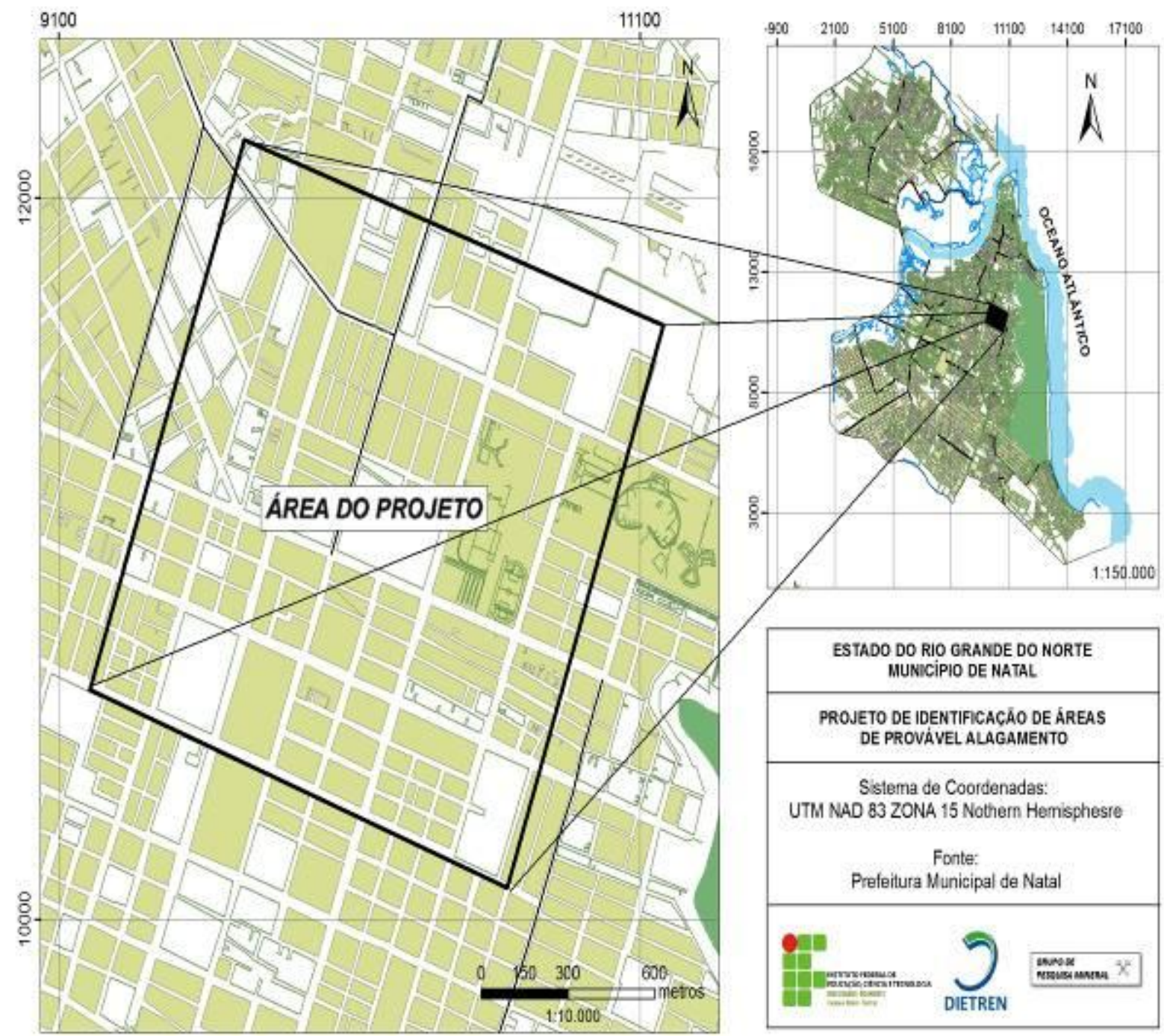

Figura 4 - Mapa de localização do trabalho 


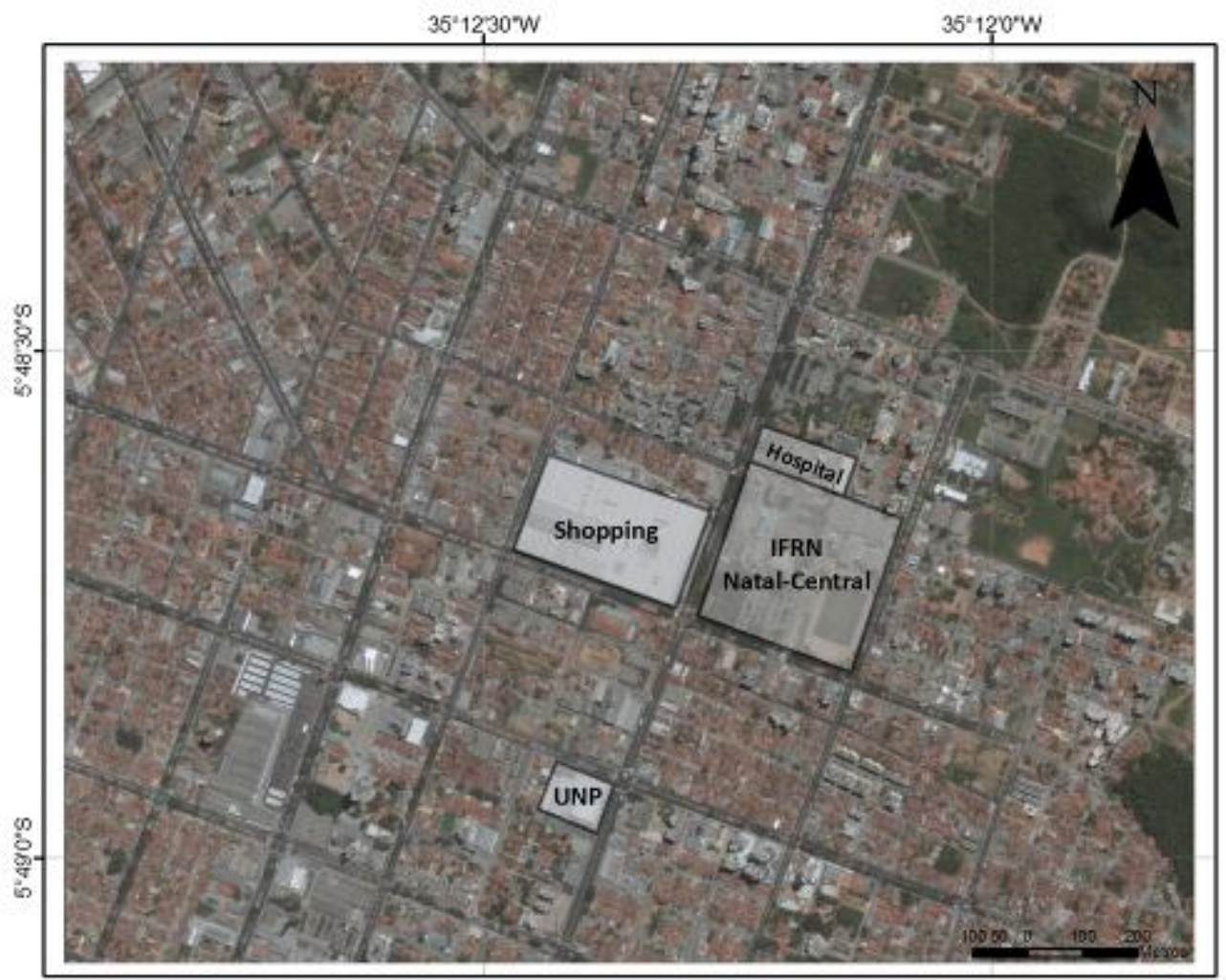

\section{Figura 5 - Fotografia aérea da área em estudo nesse trabalho com a localização de alguns pontos importantes.}

Diferentemente de outras cidades, não se identifica em Natal, no trecho enfocado neste trabalho, tragédias devido ao transbordamento de rios e/ou riachos, como registrado em São Paulo, Rio de Janeiro, etc. Aqui, as chuvas, que eram absorvidas pelas areia de dunas para alimentar o aquífero livre, agora escoam pelas ruas pavimentadas e se acumulam, junto com o lixo, em regiões de cotas baixas e sem drenagem receptora. Não é raro, locais com drenagem receptora artificial entulhada com o lixo, impermeabilizando, pelo entupimento, os bueiros e provocando os alagamentos, e consequentemente, as doenças.

Os dados da EMPARN op cit demonstram que é de extrema importância a preocupação com a drenagem na capital, atualmente com 107 pontos problemáticos, segundo dados da Prefeitura Municipal e da UFRN. Mesmo sendo um ano considerado de média pluviosidade, 2010 tem mostrado períodos difíceis em dias chuvosos para a população: o trânsito se torna caótico, pois diversas vias de circulação acabam sendo interrompidas devido às chuvas e à drenagem incapaz de escoar o volume de águas pluviais.

\section{METODOLOGIA}

Pretende-se estender esse trabalho para toda a área de Natal, no entanto, a atual fase enfoca apenas um fragmento de cena no entorno do campus central do IFRN, conforme ilustram as figuras 4 e 5 . A base dos dados, utilizada para apresentação desses dados preliminares, foi obtida através de Imagem SRTM (Shuttle Radar Topography Mission), de satélite passivos (CBERS e Google Earth), plantas da cidade de Natal e dados plani-altimétricos disponíveis. Foram utilizados os softwares Global Mapper, ArcGis 9.2 e o Microsoft Access. A checagem de campo e os dados históricos da área e registros de alagamentos (Cruz, 2010; EMPARN, 
2010; Xavier, 2006), também foram fundamentais para revalidar as interpretações de sensoriamento remoto.

Os dados de altimetria possibilitaram a elaboração de curvas de nível (figura 6), permitindonos alguns ensaios com diferentes eqüidistâncias visando escolher aquela mais adequada aos propósitos deste trabalho.

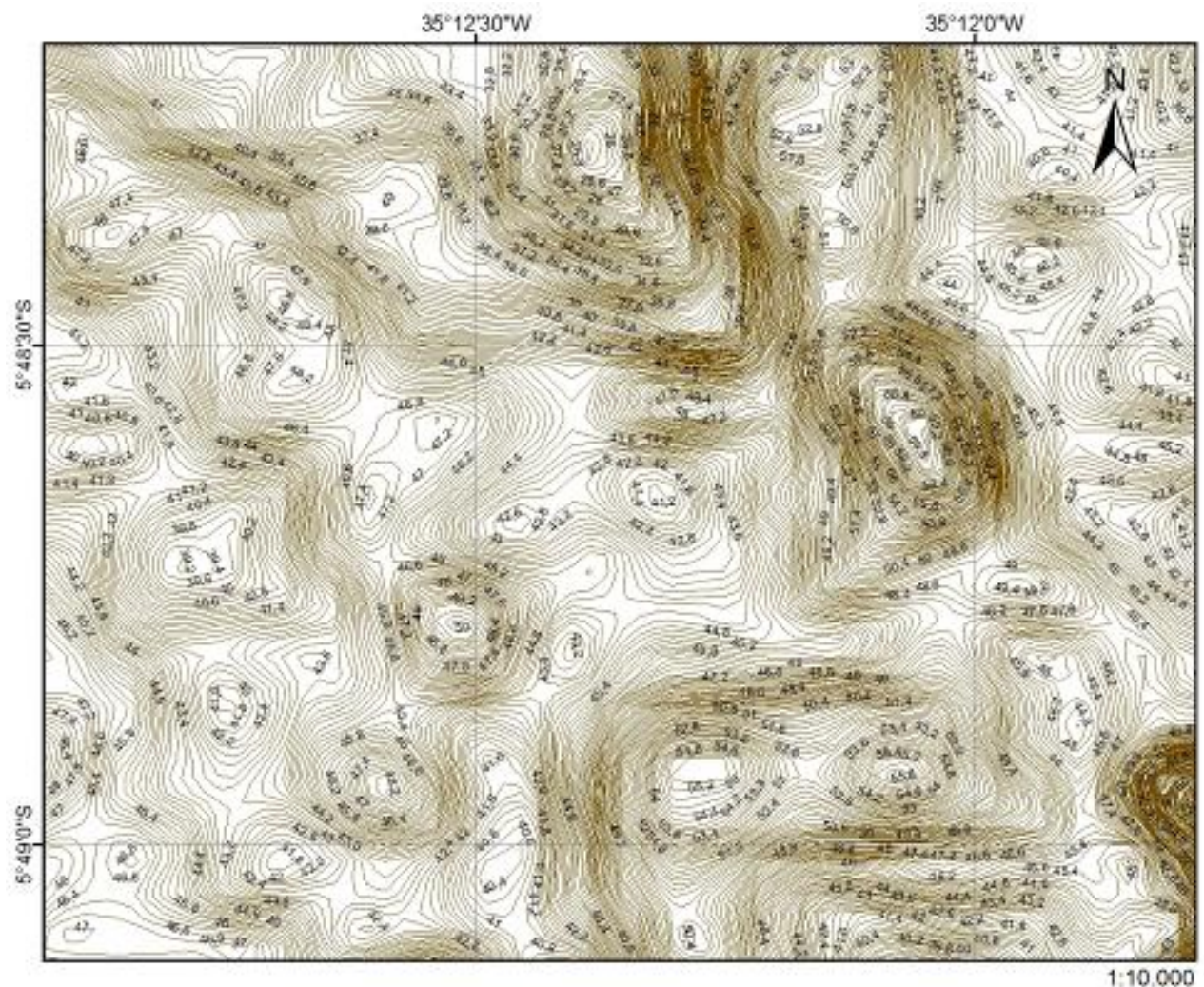

Figura 6 - Linhas de curva de nível da área

Com o Global Mapper e os dados de plani-altimetria auxiliado pelo SRTM, foi elaborado o modelo 3D reproduzido na figura 7.

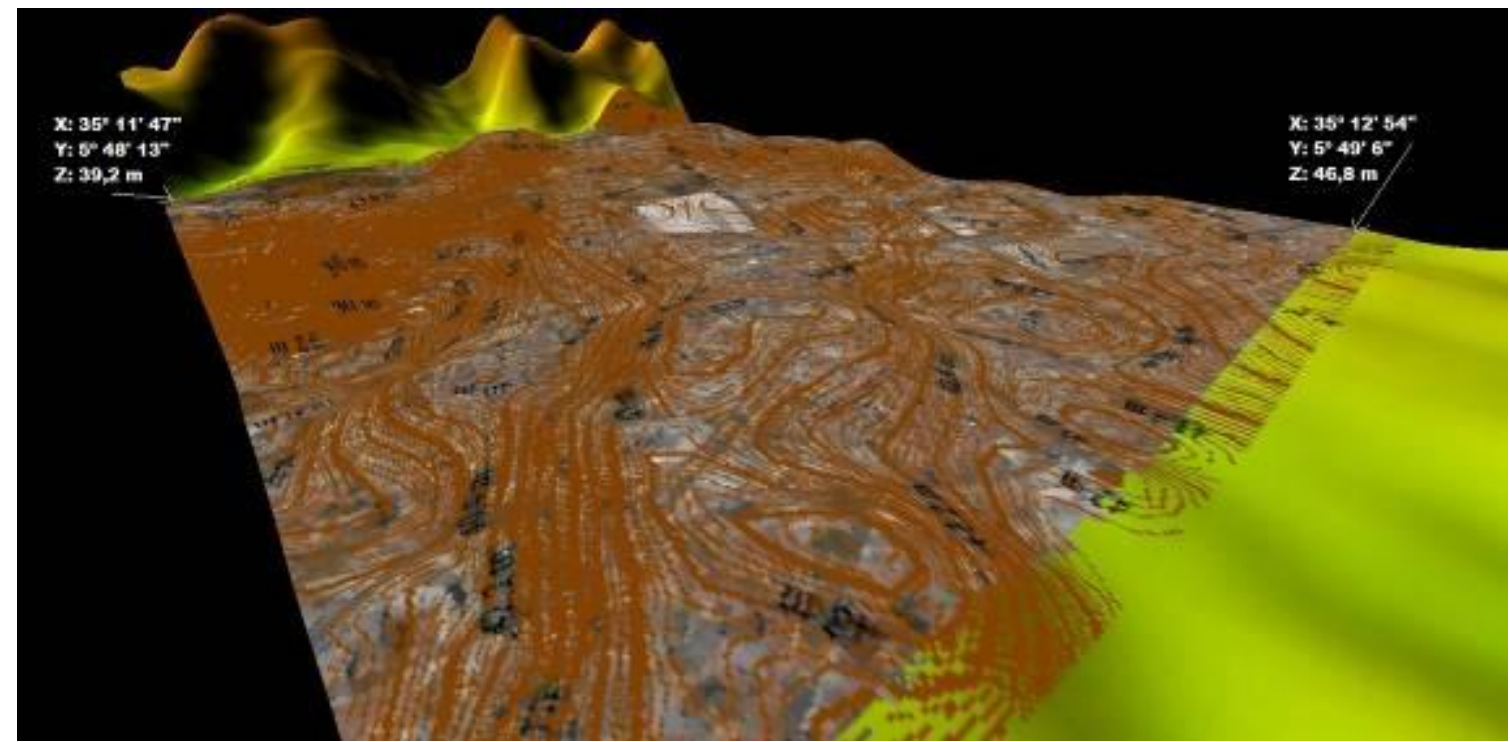

Figura 7 - Modelo 3D da área 
Juntamente com as curvas de nível, esses modelos proporcionaram a construção de Vetores de Fluxo de Drenagem (VFD), levando em consideração a altimetria e o traçado dos arruamentos observados em satélite e planta. A confluência de vetores de fluxo de drenagem (figura 8) determina os possíveis locais de vulnerabilidades ao alagamento, os quais necessitam de confirmação por observações "in loco" e entrevistas com populares.

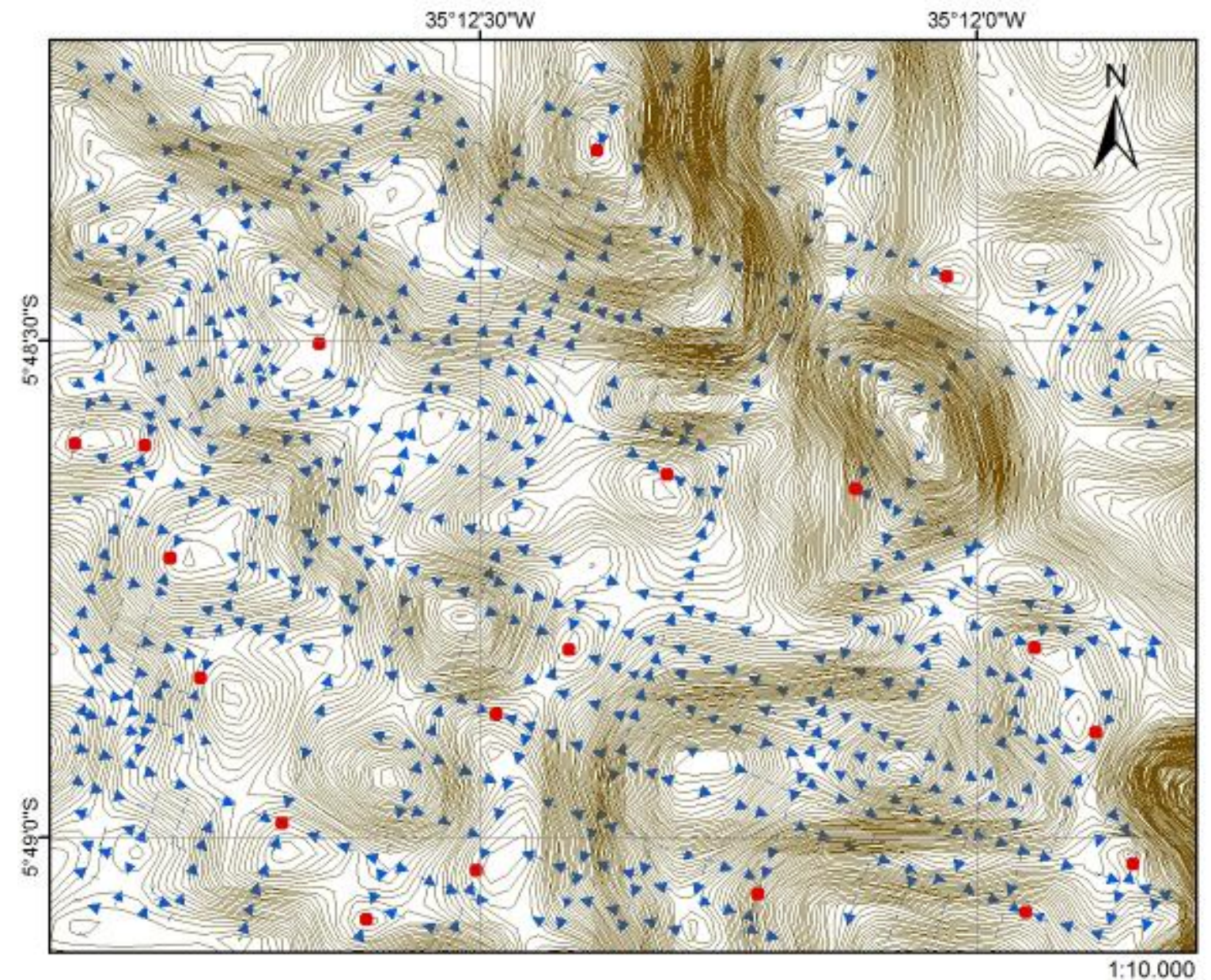

Figura 8 - Vetores de fluxo de drenagem

Com o software Global Mapper 11, simulou-se diferentes situações de alagamento a partir de mudanças da cota e espessura de lâmina d'água pluvial. Levando em consideração que a cota mínima da área é de $25 \mathrm{~m}$, foram simulados cenários de 5 e 15 metros de lâmina d'água a partir dessa cota. Essas simulações foram utilizadas para reforçar a existência de locais vulneráveis ao alagamento, caracterizados por um perfil de depressão. As áreas azuis nas figuras 9 e 10 identificam as primeiras regiões a serem alagadas na área em estudo, segundo a topografia. É importante notar que as duas simulações são caracterizadas por um nível de exagero em seus dados, visto que não há registros de alagamentos na região desse trabalho que tenha alcançado 5 metros de lâmina d'água. Portanto, o uso dessas é justificado apenas por se caracterizar numa ferramenta auxiliadora na identificação dos locais de vulnerabilidade. 


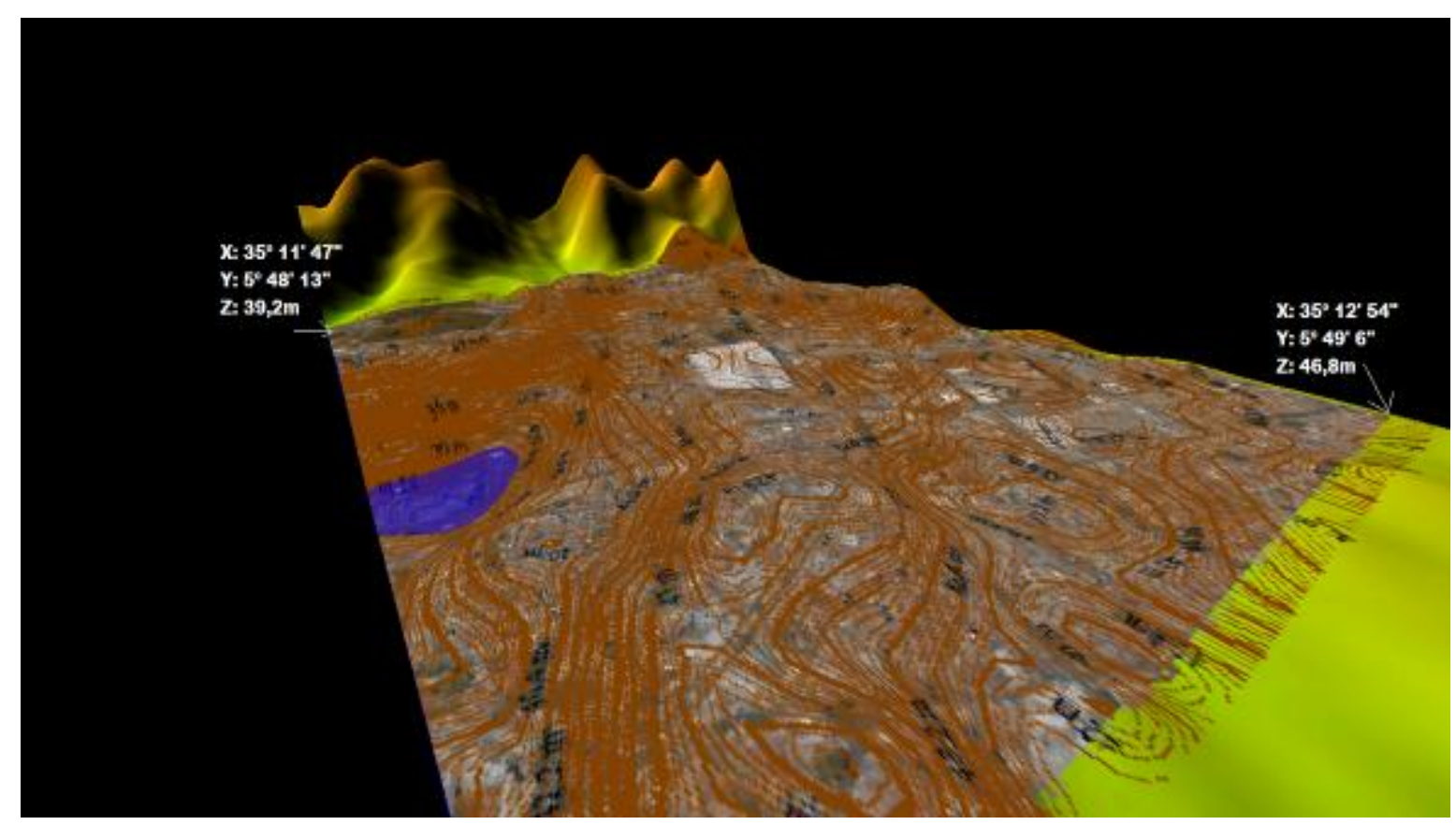

Figura 9 - Simulação: cota igual a 5 metros (de alagamento)

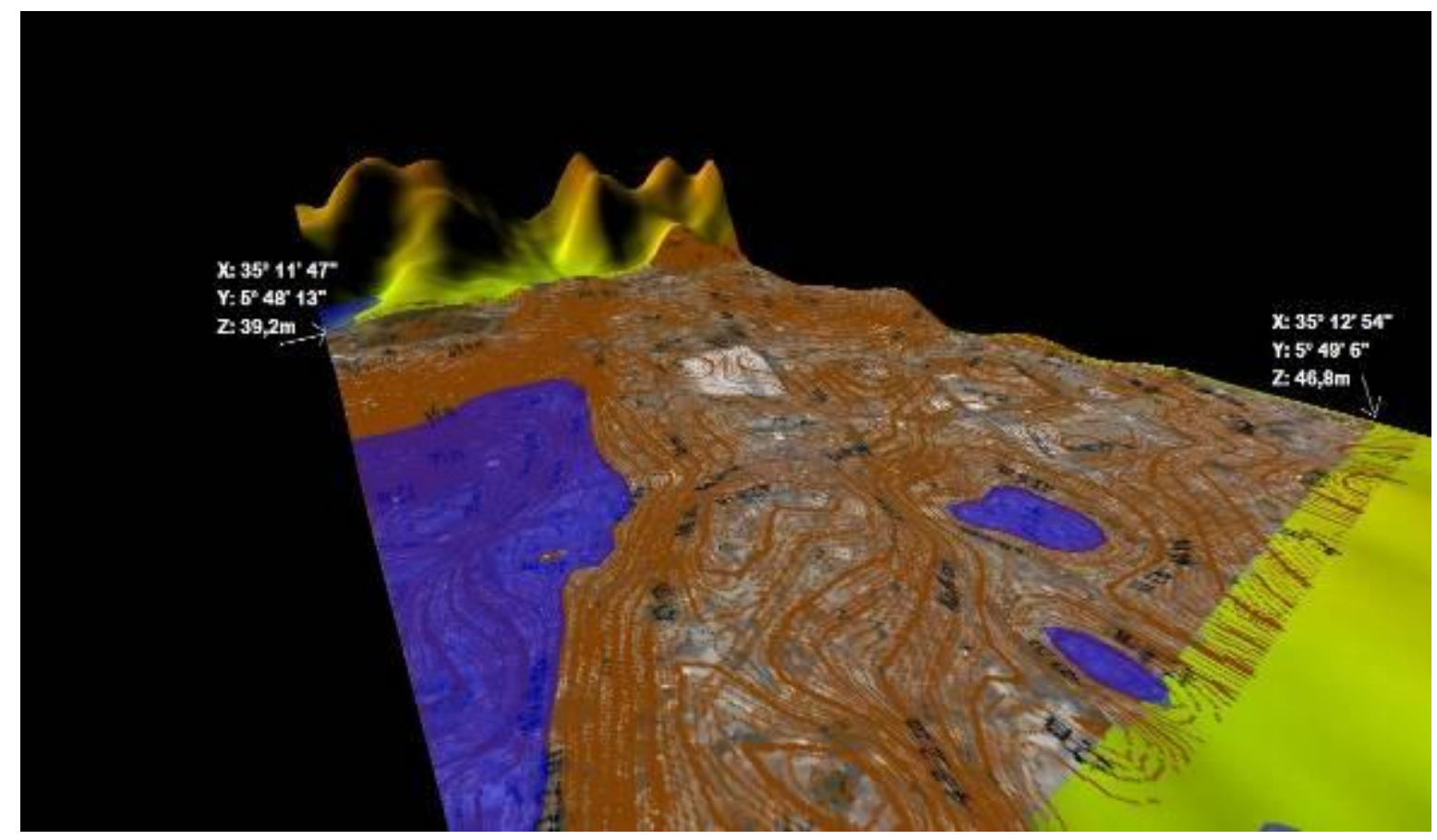

Figura 10 - Simulação: cota igual a 15 metros (de alagamento)

Foram elaborados perfis topográficos nas áreas de convergência de VDFs (figuras 11 e 12) constituindo-se em mais uma informação para apontar as áreas vulneráveis ao alagamento.

\section{RESULTADOS}


Foram observados 19 pontos vulneráveis, com localização descrita na Tabela 1, ao alagamento na área em análise, confirmados com base no histórico dos últimos anos segundo Cruz, 2010, EMPARN, 2010, e Xavier, 2006.

Tabela 1 - Descrição dos pontos identificados como vulneráveis ao alagamento.

\begin{tabular}{|c|c|c|}
\hline CÓDIGO & LOGRADOURO & $\begin{array}{l}\text { COORDENADAS } \\
(\mathrm{X}, \mathrm{Y}) \text { WGS } 1984\end{array}$ \\
\hline ALAG-NAT-01/01 & R. Dr. Múcio Galvão & $\begin{array}{c}35^{\circ} 12^{\prime}, 23^{\prime \prime}, \mathrm{W} \\
5^{\circ} 48^{\prime} \\
19^{\prime}, \mathrm{S}\end{array}$ \\
\hline ALAG-NAT-01/02 & R. Mal. Mallet & $\begin{array}{c}35^{\circ} 12^{\prime} 1^{\prime \prime} \mathrm{W} \\
5^{\circ} 48^{\prime} 26^{\prime \prime} \mathrm{S}\end{array}$ \\
\hline ALAG-NAT-01/03 & R. Cel. Silvino Bezerra & $\begin{array}{c}35^{\circ} 12^{\prime} 39^{\prime \prime} \mathrm{W} \\
5^{\circ} 48^{\prime} 30^{\prime \prime} \mathrm{S}\end{array}$ \\
\hline ALAG-NAT-01/04 & Av. Jaguarari & $\begin{array}{c}35^{\circ} 12^{\prime} 54^{\prime \prime} \mathrm{W} \\
5^{\circ} 48^{\prime} 36^{\prime}, \mathrm{S}\end{array}$ \\
\hline ALAG-NAT-01/05 & R. Dr. Horácio & $\begin{array}{c}35^{\circ} 12^{\prime}, 50^{\prime \prime} \mathrm{W} \\
5^{\circ} 48^{\prime} 37^{\prime \prime} \mathrm{S}\end{array}$ \\
\hline ALAG-NAT-01/06 & R. São Joaquim & $\begin{array}{c}35^{\circ} 12^{\prime} 18^{\prime \prime}, \mathrm{W} \\
5^{\circ} 48^{\prime} 38^{\prime \prime} \mathrm{S}\end{array}$ \\
\hline ALAG-NAT-01/07 & R. Ten. Júlio Gomes & $\begin{array}{c}35^{\circ} 12^{\prime} 7^{\prime \prime} \mathrm{W} \\
5^{\circ} 48^{\prime} 39^{\prime \prime} \mathrm{S}\end{array}$ \\
\hline ALAG-NAT-01/08 & R. da Aurora & $\begin{array}{c}35^{\circ} 12^{\prime} 48^{\prime \prime} \mathrm{W} \\
5^{\circ} 48^{\prime} 43^{\prime}, \mathrm{S}\end{array}$ \\
\hline ALAG-NAT-01/09 & R. Cussy & $\begin{array}{c}35^{\circ} 12^{\prime} 24^{\prime \prime} \mathrm{W} \\
5^{\circ} 48^{\prime} 48^{\prime \prime} \mathrm{S}\end{array}$ \\
\hline ALAG-NAT-01/10 & Av. Xavier da Silveira & $\begin{array}{c}35^{\circ} 11^{\prime} 56^{\prime \prime} \mathrm{W} \\
5^{\circ} 48^{\prime} 48^{\prime}, \mathrm{S}\end{array}$ \\
\hline ALAG-NAT-01/11 & R. São José & $\begin{array}{c}35^{\circ} 12^{\prime} 46^{\prime \prime} \mathrm{W} \\
5^{\circ} 48^{\prime} 50^{\prime}, \mathrm{S}\end{array}$ \\
\hline ALAG-NAT-01/12 & Av. Antonio Basílio & $\begin{array}{c}35^{\circ} 12^{\prime} 28^{\prime \prime}, \mathrm{W} \\
5^{\circ} 48^{\prime} 52^{\prime}, \mathrm{S}\end{array}$ \\
\hline ALAG-NAT-01/13 & R. da Torre & $\begin{array}{c}35^{\circ} 11^{\prime} 52^{\prime \prime}, \mathrm{W} \\
5^{\circ} 48^{\prime} 53^{\prime \prime} \mathrm{S}\end{array}$ \\
\hline ALAG-NAT-01/14 & Av. Prudente de Morais & $\begin{array}{c}35^{\circ} 12^{\prime} 41^{\prime \prime} \mathrm{W} \\
5^{\circ} 48^{\prime} 59^{\prime}, \mathrm{S}\end{array}$ \\
\hline ALAG-NAT-01/15 & R. Dr. José Gonçalves & $\begin{array}{c}35^{\circ} 12^{\prime} 30^{\prime \prime} \mathrm{W} \\
5^{\circ} 49^{\prime} 02^{\prime}, \mathrm{S}\end{array}$ \\
\hline ALAG-NAT-01/16 & Av. Djama Maranhão & $\begin{array}{c}35^{\circ} 11^{\prime} 50^{\prime \prime} \mathrm{W} \\
5^{\circ} 49^{\prime} 01^{\prime}, \mathrm{S}\end{array}$ \\
\hline ALAG-NAT-01/17 & Av. Romualdo Galvão & $\begin{array}{c}35^{\circ} 12^{\prime} 36^{\prime \prime} \mathrm{W} \\
5^{\circ} 49^{\prime} 05^{\prime}, \mathrm{S}\end{array}$ \\
\hline
\end{tabular}




\begin{tabular}{|c|c|c|}
\hline ALAG-NAT-01/18 & Av. Rui Barbora & $35^{\circ} 12^{\prime} 13^{\prime}, \mathrm{W}$ \\
& & $5^{\circ} 49^{\prime} 03^{\prime}, \mathrm{S}$ \\
\hline ALAG-NAT-01/19 & Av. Antonio Basílio & $35^{\circ} 11,57^{\prime}, \mathrm{W}$ \\
& & $5^{\circ} 49^{\prime} 04$ ', $\mathrm{S}$ \\
\hline
\end{tabular}

A figura 11 mostra a localização dos pontos identificados através da interpretação da topografia e dos VFDs, como mostrados na figura 8.

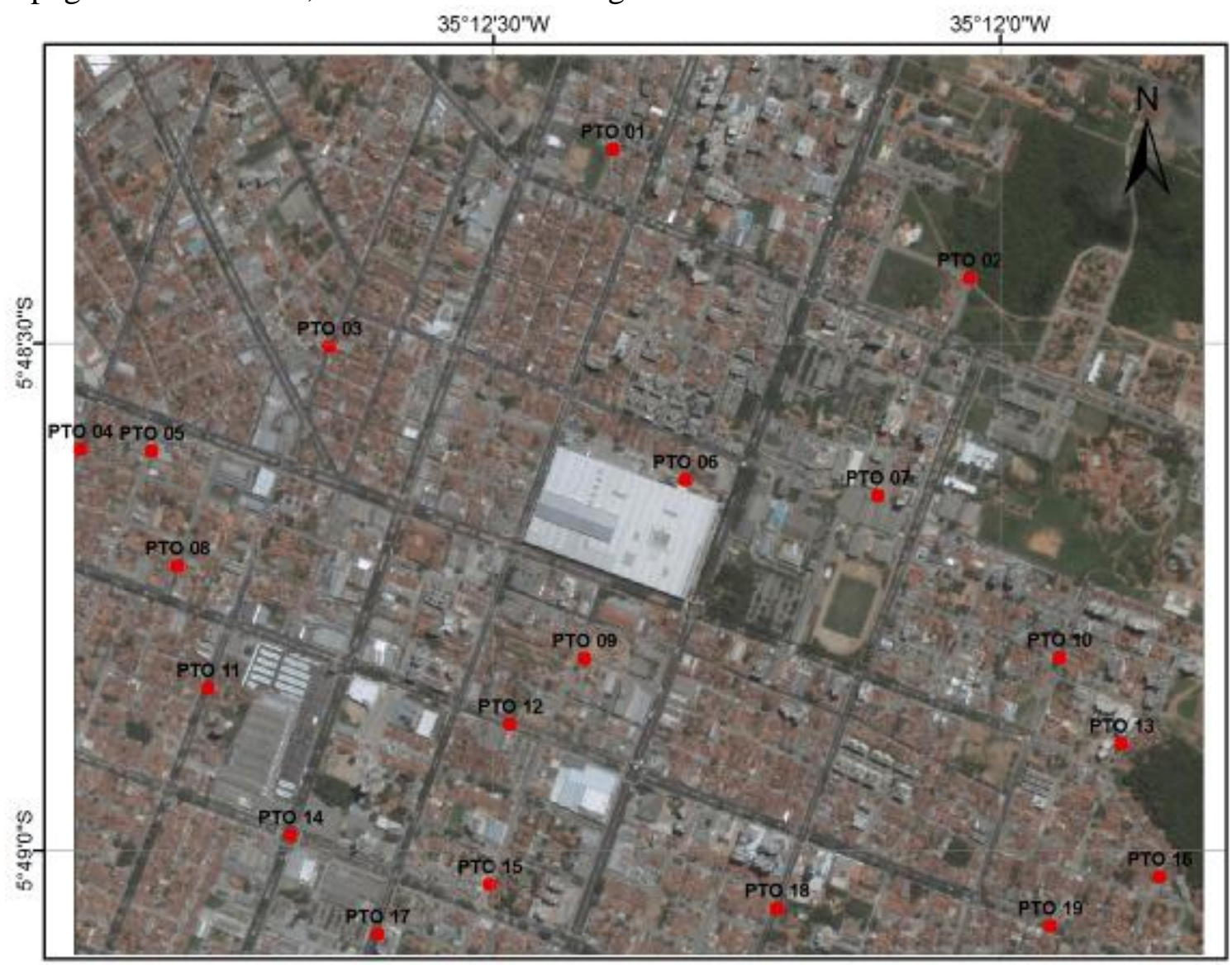

$1: 10.000$

Figura 11 - Localização dos pontos vulneráveis ao alagamento.

Nas figuras 12 e 13, os perfis topográficos da rua Dr. Múcio Galvão (ponto ALAG-NAT01/01), nas proximidades do supermercado Bompreço e do Fast Food Mc Donald's, e da av. Xavier da Silveira (ponto ALAG-NAT-01/10), utilizados como exemplos, exibem uma configuração geométrica côncava, onde o estrangulamento no escoamento superficial não permite a saída da água no mesmo fluxo de entrada nos períodos chuvosos, ou em chuvas excepcionais de média ou elevada magnitude causando transtorno à população. Essa geometria, identificada em todos os pontos descritos na Tabela 1 permite a afirmação de que tais locais são vulneráveis ao alagamento. 
From Pos: -35.2059272871, -5.To Pos: -35.2066373683, -5.8059596640

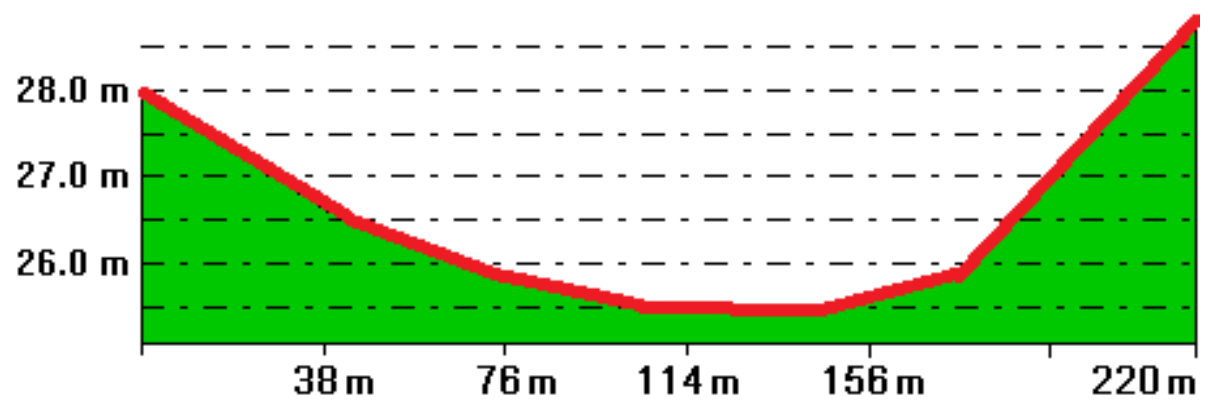

Figura 12 - Perfil de ponto vulnerável, rua Dr. Múcio Galvão

From Pos: -35.1994229176, -5.To Pos: -35.1985864515, -5.8123522893

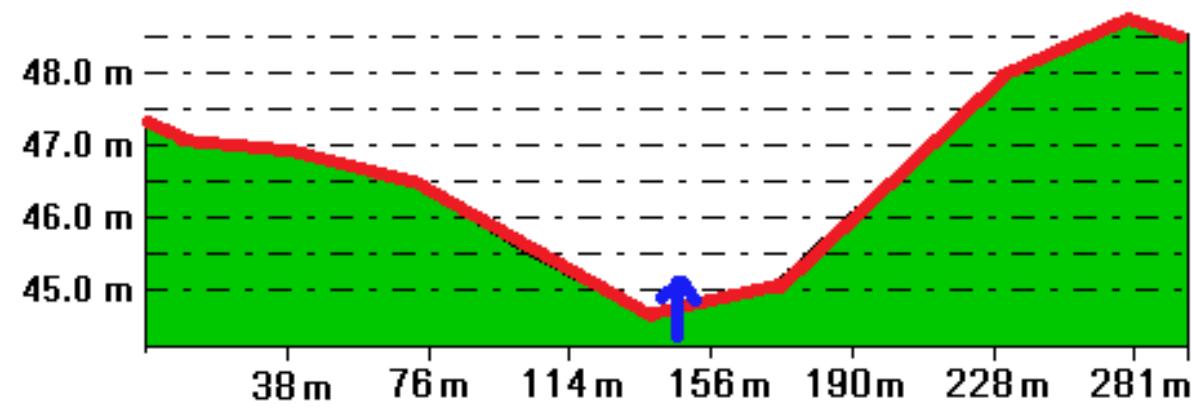

Figura 13 - Perfil de ponto vulnerável, cruzamento da rua Eng. Antônio Lira (seta azul) com av. Xavier da Silveira (linha vermelha)

É importante notar nos perfis das figuras 12 e 13 a diferença de cota dos perfis, variando de 2 a 3 metros nos exemplos supracitados, dado esse que caracteriza ambos os pontos como dois dos mais críticos, junto à rua São Joaquim (ponto ALAG-NAT-01/06). Para a análise dos dados, é indispensável a observação do fluxo, suas vias distribuidoras e coletoras. No caso da figura 14, observa-se que a rua atrás do campus do IFRN é uma via distribuidora de água para a Avenida Bernardo Vieira, que a cruza. Essa geometria favorece a distribuição da água por outras vias, como a Avenida Senador Salgado Filho e por outras até que ocorra o alagamento em vias coletoras.

From Pos: 5" 48' 58.9014' S, 35"12' 11.6922"'W

To Pos: 5" 48' 31.3215" S, 35"12' 1.5698"'W

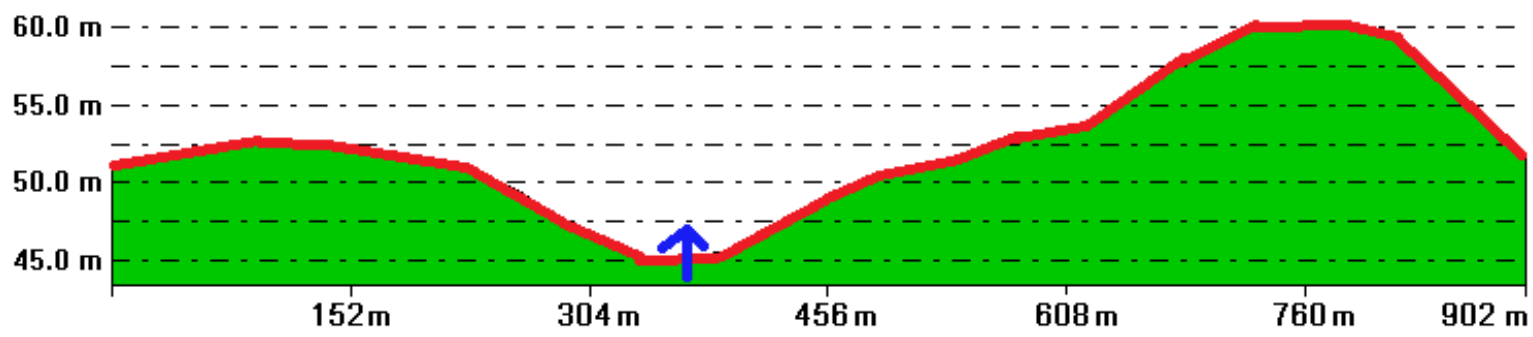

Figura 14 - Perfil topográfico da rua Zacarias Monteiro (linha vermelha) com cruzamento da av. Bernardo Vieira (seta azul)

\section{CONSIDERAÇÕES FINAIS}

Um evento calamitoso só ocorre quando há uma união entre um agente (ameaça) e uma vulnerabilidade (fraqueza) (Cortez, 2003). Na cidade de Natal, a vulnerabilidade está no alto nível de impermeabilização do solo aliado ao sistema de drenagem insuficiente e/ou 
inexistente em diversos pontos, acrescido da falta de manutenção dos sistemas de drenagens artificiais, lixo que causa entupimentos, falta de saneamento básico e as ligações clandestinas de esgotos. Todos esses fatos não apenas são geradores de risco de alagamento, mas acentuam os problemas de saúde.

No caso dos pontos identificados por esse trabalho, os fatores que causam os alagamentos podem ser generalizados: topografia favorável, com geometria côncava em vias coletoras de fluxo, e impermeabilização do solo, com número expressivo de cobertura pavimentada e pouca área permeável.

Na capital do Estado do Rio Grande do Norte os alagamentos são provocados pelas águas pluviais que ocorrem mais intensamente entre os meses de março e agosto. Os alagamentos são problemas de ordem socioeconômica e ambiental, pois impedem o funcionamento normal das atividades da sociedade e causam prejuízos de toda ordem, inclusive financeiro. A cidade se rende ao caos provocado por essa problemática, cujas consequências vão além dos prejuízos financeiros, às limitações do ir e vir da população e aumento de doenças e epidemias.

A continuação deste trabalho, e na dependência de financiamentos, pretende-se fazer este levantamento em toda a cidade do Natal.

\section{REFERÊNCIAS}

1. CORTEZ, Henrique. Riscos Ambientais Urbanos. Revista Mais Brasil, novembro/2003.

2. CRUZ, Carla. Chuvas: Natal tem 72 pontos de alagamento. Portal Nominuto.com. Disponível em: <http://www.nominuto.com/noticias/cidades/chuvas-natal-tem-72pontos-de-alagamento/51060/>. Acesso em: 2 jul 2010

3. EMPARN. Pluviometria da cidade de Natal - RN. Disponível em: <http://www.emparn.rn.gov.br/contentproducao/aplicacao/emparn/arquivos/meteor ologia/analise_chuva.asp> Acesso em: 4 jul 2010.

4. MANUAL DE DESASTRES. Desastres Naturais - vol. I. Ministério da Integração Nacional, Secretaria Nacional de Defesa Civil. Brasília-DF, 2003.

5. SANTOS, R. F. (org). Vulnerabilidade Ambiental - Desastres Naturais ou Fenômenos Induzidos? Brasília: MMA, 2007. 192p.

6. SILVEIRA, Patrícia Gomes da; BESER DE DEUS, Leandro Andrei; SANTOS, Camilla Silva Motta. Análise espacial de áreas afetadas por eventos hidrológicos extremos na cidade do Rio de Janeiro. UFRJ, Rio de Janeiro, 2008.

7. SOBREIRA, Liése Carneiro; ROSA, Paulo Roberto de Oliveira; ROSA, Conrad Rodrigues; NASCIMENTO, Maria Odete T.; PAZERA Jr., Eduardo. Distribuição das áreas de inundação na cidade de João Pessoa, Paraíba: esboço de análise geográfica. In: CONGRESSO BRASILEIRO DE GEÓGRAFOS, 6, Goiana, Anais... Goiás: UFG, 2004. CD-ROM. 
8. WACCHOLZ, Flávio; PEREIRA Filho, Waterloo. Mapeamento do uso da terra na bacia hidrográfica do arroio Barriga - RS, utilizando o sensoriamento remoto. In: Jornada de Educação em Sensoriamento Remoto no Âmbito do Mercosul, 4.. 2004. São Leopoldo. Anais... São Leopoldo, 2004.

9. XAVIER, Michele Mota. Riacho do Baldo: às margens da cidade? 2006. Monografia (Graduação em Arquitetura e Urbanismo) - Universidade Federal do Rio Grande do Norte, 